\title{
Mechanical Properties of Gangue Cement Stabilized Road Foundation Mixture
}

\author{
Dongsheng Zhang ${ }^{1}$, Naicheng Liu ${ }^{2}$, Mingjie Mao ${ }^{2 *}$, Jiabin Li $^{1}$ \\ ${ }^{1}$ Research Group RecyCon, Department of Civil Engineering, KU Leuven, Campus Bruges, 8200, Bruges, Belgium \\ ${ }^{2}$ School of Civil and Hydraulic Engineering, Ningxia University, Yinchuan 750021, China
}

\begin{abstract}
In this study, coal gangue was used to replace the four single-graded natural gravels of $0 \sim 5 \mathrm{~mm}$, $5 \sim 10 \mathrm{~mm}, 10 \sim 20 \mathrm{~mm}$, and $20 \sim 26.5 \mathrm{~mm}$ to design the mix ratio and to study the influence in particle size changes of coal gangue on the mechanical properties of the mixture. The research results show that: The maximum dry density of the mixture with coal gangue is lower than the density of the natural crushed stone water-stable mixture, and the optimal moisture content is higher than that of natural crushed stone waterstable mixture; coal gangue aggregate is mixed with cement stabilized in the crushed stone mixture, and the $7 \mathrm{~d}$ and $28 \mathrm{~d}$ unconfined compressive strength, splitting strength, and compression rebound modulus values of the mixture are all reduced, which has a degrading effect on the mechanical properties of the mixture; however, both can attain high speed, which is the basic strength requirements for expressways, including first-class highways.
\end{abstract}

\section{Introduction}

In China, coal is the major energy source. Coal consumes $70 \%$ of its primary energy, which is more than twice the world average[1]. Most of the coal gangue is stacked directly after it is produced. The gangue piles stored in the open air for a long time can easily and spontaneously combust and produce harmful gases, and the weathered coal gangue thus enters the surrounding soil, air, and groundwater, causing pollution. Therefore, recycling of coal gangue is conducive to protecting the environment and restoring the natural ecology[2].

In civil engineering, recycled coal gangue is mostly used as an aggregate for road water-stable layers. First, highway engineering does not have stringent requirements for the type and quality of gangue, and most of the gangue meets engineering construction standards. Second, coal gangue, instead of natural crushed stone aggregate, does not require special technical treatment and only requires a suitable crushing and screening method[2].

Therefore, based on ordinary cement-stabilized crushed stone base materials, referring to the index requirements proposed in the Chinese standard, this study uses coal gangue instead of different grading particle sizes to influence the mechanical properties of the mixture and aims to develop a suitable Ningxia. The coal gangue base material not only alleviates the shortage of natural aggregates but also efficiently uses coal gangue materials. This conforms to the technical principles of localization of road construction materials and converts waste into valuable material.

\section{Raw materials and experimental plan}

\subsection{Raw materials}

Cement: Ningxia Saima brand PO42.5 ordinary Portland cement was used; For natural gravel, this study used the natural limestone of Ningxia Taomengou Stone Material Factory and based on the "Highway Pavement Base Construction Technical Regulations" ; four grades of aggregates were used, divided into grades of 0-5 mm, 5$10 \mathrm{~mm}, 10-20 \mathrm{~mm}$, and 20-26.5 mm. For coal gangue, this test uses coal gangue discharged from coal mines in Ningxia, all of which are crushed and screened using jaw crushers and divided into four categories: 0-5 mm, 5-10 $\mathrm{mm}, 10-20 \mathrm{~mm}$, and 20-26.5 mm gradation; furthermore, the Yinchuan city tap water was used.

\subsection{Experimental program}

The cement content was found to be $4 \%$. Based on the screening results, the particle size of natural crushed stone was found to be $20-26.5 \mathrm{~mm}$ : $10-20 \mathrm{~mm}$ : 5-10 mm: 0-5 $\mathrm{mm}$, and the ratio was 0.18: 0.275: 0.18: 0.365 , respectively. The cement-stabilized crushed stone mixture is denoted as $\mathrm{M} 0$, the mixture using $0-5 \mathrm{~mm}$ coal gangue aggregate particle size is denoted as $\mathrm{M}(0-5)$, and that with 5-10 $\mathrm{mm}$ coal gangue aggregate particle size is denoted as $\mathrm{M}(5-10)$. The mixture with $10-15 \mathrm{~mm}$ coal gangue aggregate particle size was recorded as M (10-20), and that with $20-26.5 \mathrm{~mm}$ coal gangue aggregate particle size was

\footnotetext{
* Corresponding author: maomjnxdx@163.com
} 
recorded as M (20-26.5). In the experiment, a heavy-duty compaction test determined the optimal moisture content and dry density index of the material.

The test piece of the mixture was made using a singlehorizontal shaft forced mixer to stir the mixture. The mixing sequence was first pour the stabilized material into the mixer and stir for $30 \mathrm{~s}$ for uniform gradation; subsequently, pre-weighed water was added and stirred for $60 \mathrm{~s}$. Finally, a container was used to slowly add cement and stirred for $120 \mathrm{~s}$ to evenly combine the stabilized material and the cement. Based on the static pressure forming method in the "Highway Engineering Inorganic Binder Stabilizing Material Test Regulations" (JTG E51-2009)[3], a WYC-150 II stabilized soil specimen-forming machine was used to form a diameter of $150 \mathrm{~mm}$ and a diameter-to-height ratio of $1: 1$; the compaction coefficient of the cylindrical specimen was 0.98 , and the specimen was wrapped tightly in a plastic bag and cured in an environment with a temperature of $20 \pm 2{ }^{\circ} \mathrm{C}$ and a humidity of $\geq 95 \%$. The specimen was immersed in water for one day before reaching its age[4]. The unconfined compressive strength, split strength, and compressive resilience modulus tests were conducted after the specimens reached the ages of $7 \mathrm{~d}$ and $28 \mathrm{~d}$.

\section{Results and discussion}

\subsection{Maximum dry density and optimal moisture content}

Based on the "Technical Regulations for Highway Pavement Base Construction," the test pieces formed by the heavy compaction method were used to determine the optimum moisture content and maximum dry density of the mixture of coal gangue with different alternative particle sizes. The test results are presented in Table 1.

Table1. The optimal moisture content and maximum dry density of the mixture

\begin{tabular}{ccc}
\hline Test group & $\begin{array}{c}\text { maximum dry } \\
\text { density }\left(\mathrm{g} / \mathrm{cm}^{3}\right)\end{array}$ & $\begin{array}{c}\text { optimal moisture } \\
\text { content }(\%)\end{array}$ \\
\hline $\mathrm{M} 0$ & 2.325 & 4.90 \\
$\mathrm{M}(0-5)$ & 2.268 & 5.13 \\
$\mathrm{M}(5-10)$ & 2.292 & 5.01 \\
$\mathrm{M}(10-20)$ & 2.252 & 5.23 \\
$\mathrm{M}(20-26.5)$ & 2.250 & 5.06 \\
\hline
\end{tabular}

From Table 1, the maximum dry density of the coal gangue cement-stabilized mixture is lower than that of the natural crushed stone water-stable mixture, and the optimal moisture content is higher than that of the natural crushed stone water-stable mixture, which is similar to that of the coal gangue. Density and water absorption are related. The density of coal gangue is lower than that of natural gravel; however, the water absorption is higher. Using 0-5 mm coal gangue as an example, compared with natural gravel, the maximum dry density of the coal gangue cement-stabilized mixture was reduced by $2.45 \%$, and the optimal moisture content was increased by $4.7 \%$. This shows that the incorporation of coal gangue has varying effects on the maximum dry density and optimal moisture content of the mixture. In physical engineering, various technical parameters should be strictly controlled to reduce the risk of cracks owing to the increase in the moisture content of the mixture[5].

\subsection{Unconfined compressive strength}

The unconfined compressive strength test of the $7 \mathrm{~d}$ and $28 \mathrm{~d}$ aged mixture of coal gangue, instead of crushed stones of different single particle size, was conducted, and it was combined with cement stabilized crushed stone mixture benchmark group (M0 group) and cement stabilized coal gangue mixture (M100 group) For comparison, the representative values of unconfined compressive strength and coefficient of variation are shown in Figures 1 and 2, respectively.

From Figure 1, compared with the natural crushed stone mixture benchmark group M0, the unconfined compressive strength of the $7 \mathrm{~d}$ mixture of coal gangue instead of natural crushed stone of different particle sizes demonstrated different degrees of decline, M(0 -5), M (510), $M(10-20)$, and $M(20-26.5)$ groups, and the $7 d$ unconfined compressive strength decreased by $54.2 \%$, $39.0 \%, 52.3 \%$, and $42.0 \%$, respectively; that is, the substitution of $0-5 \mathrm{~mm}$ and $10-20 \mathrm{~mm}$ coal gangue coal has a greater impact on the strength of the material. In addition, with the increase in age to $28 \mathrm{~d}$, the unconfined compressive strength of each ratio increased, including $\mathrm{M}(0-5), \mathrm{M}(5-10), \mathrm{M}(10-20)$, and M(20- 26.5). Compared with the intensity of $7 \mathrm{~d}$, the intensity increased by $16.6 \%$, $9.4 \%, 20.9 \%$, and $12.5 \%$ respectively.

Figure 2 shows that the replacement of natural gravel with coal gangue generally increases the dispersion of the test results of the mixture. At $7 d$, the coefficient of variation of the $\mathrm{M}(0-5)$ mixture was lower than that of the natural crushed stone mixture benchmark group M0, whereas for the other groups, it still improved to varying degrees. At $28 \mathrm{~d}$, the coefficients of variation of all coal gangue cement mixtures were much higher than those of the benchmark group, and the $M$ (0-5) group had the smallest increase in mixtures. This shows that replacing the finer natural gravel can slightly weaken the influence of coal gangue on the mechanical properties.

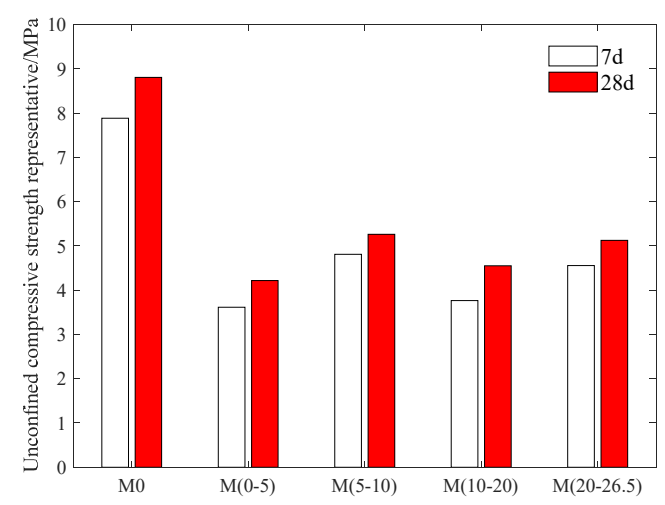

Figure 1. Unconfined compressive strength Representative of cement-stabilized coal gangue mixture 


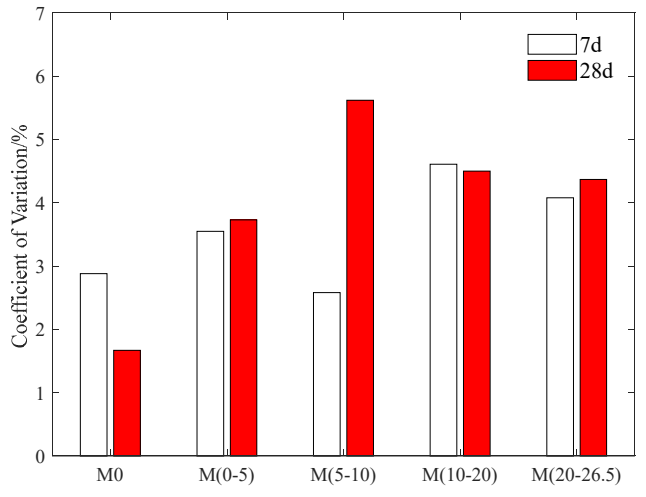

Figure 2. Coefficient of variation of cement stabilized coal gangue mixture

From the perspective of the strength increase of the composite sample with the age and the strength loss of the benchmark group, the strength of the M (5-10) and M (2026.5) groups was formed quickly, and the strength of the coal gangue was replaced. The loss is relatively low, and the unconfined compressive strength performance is better, which can meet the unconfined compressive strength requirements of the base layer under heavy traffic load conditions of expressways and first-class highways. The substitution of 0-5 $\mathrm{mm}$ gravel and 10-20 $\mathrm{mm}$ gravel has a greater impact on the unconfined compressive strength of the material[6]. The combination of $M(0-5)$ and $M(10-$ 20) can meet the strength requirements under medium and light traffic conditions.

\subsection{Splitting strength}

The $7 \mathrm{~d}$ and $28 \mathrm{~d}$ age splitting strength tests were conducted on a mixture of coal gangue instead of crushed stones of different single-particle sizes and combined with a cement-stabilized crushed stone mixture benchmark group (M0 group), cement stabilized coal gangue mixture (M100 group ), and the results of the representative values of splitting strength and the coefficient of variation are shown in Figures 3 and 4.

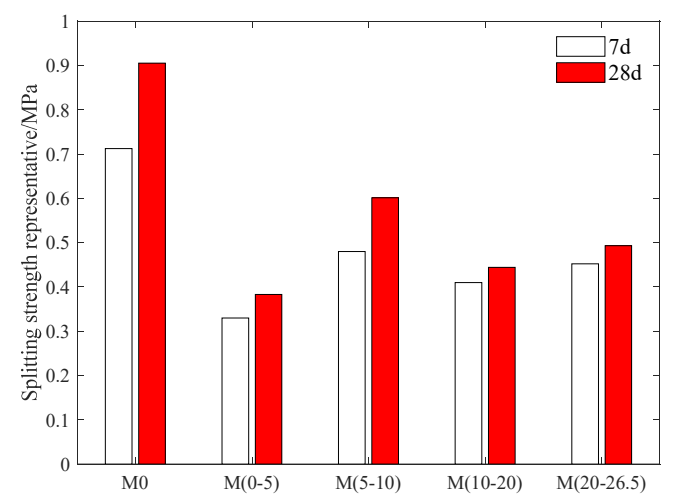

Figure 3. Splitting strength Representative of cementstabilized coal gangue mixture

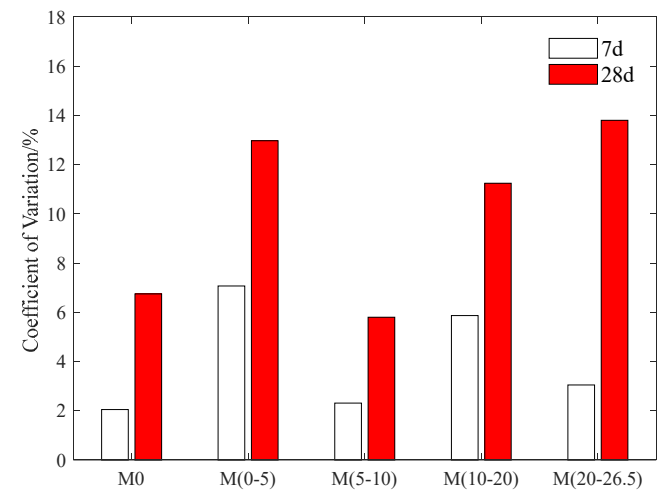

Figure 4. Coefficient of variation of cement-stabilized coal gangue mixture

From Figure 3, compared with the natural crushed stone mixture benchmark group $\mathrm{M} 0$, the $7 \mathrm{~d}$ splitting strength of the mixture of coal gangue instead of natural crushed stone of different single-particle sizes decreases to varying degrees, $\mathrm{M}(0-5)$ Compared with the benchmark group M0, the 7-d unconfined compressive strength of the, $\mathrm{M}(5-10), \mathrm{M}(10-20)$, and M (20-26.5) groups decreased by $53.65 \%, 32.58 \%, 42.42 \%$, and $36.52 \%$, respectively. The law of unconfined compressive strength is the same. The substitution of $0-5 \mathrm{~mm}$ and $10-20 \mathrm{~mm}$ coal gangue has a greater influence on the strength of the material. In addition, with the increase in age, the $28 \mathrm{~d}$ splitting strength of each ratio increases, including $\mathrm{M}(0-5), \mathrm{M}(5-$ 10), $M(10-20)$, and $M(20-26.5)$. The $7 d$ intensity increased by $16.1 \%, 25.2 \%, 8.3 \%$, and $9.1 \%$ respectively.

Figure 4 shows that the substitution of coal gangue for natural gravel generally increases the dispersion of the mixture test results. At $7 \mathrm{~d}$, the coefficient of variation of the $M(0-5)$ mixture was the lowest except for the benchmark group. Even at $28 \mathrm{~d}$, the coefficient of variation of the $\mathrm{M}(0-5)$ mixture was lower than that of the natural gravel mixture benchmark group M0. Other groups still have different degrees of improvement. It still shows that replacing the finer natural gravel can slightly weaken the influence of coal gangue on the mechanical properties.

With the combination of the strength increase of the mixture specimen during the age and the strength loss compared with the reference group, the strength of the $M$ (5-10) and M (20-26.5) groups was quickly formed, and that of the coal gangue was replaced. The loss is relatively low, and the unconfined compressive strength performance is better, which can meet the unconfined compressive strength requirements of the base layer under heavy traffic load conditions of expressways and firstclass highways; after long-term maintenance, the substitution of $0-5 \mathrm{~mm}$ and $10-20 \mathrm{~mm}$ coal gangue has a greater impact on the unconfined compressive strength of the mixture. The $\mathrm{M}(0-5)$ and $\mathrm{M}(10-20)$ groups can meet the strength requirements under medium and light traffic conditions.

In practical applications, the quality standards of coal gangue with particle sizes of 0-5 mm and 10-20 mm should be strictly controlled, or these sizes should not be used to replace natural gravel, and the splitting strength of 
the mixture should be avoided as much as possible to prevent cracks.

\subsection{Compression rebound modulus}

The compression rebound modulus representative at $7 d$ and $28 \mathrm{~d}$ for the mixture of coal gangue instead of crushed stone of different single-particle sizes, the cementstabilized crushed stone mixture benchmark group (M0 group), and the cement-stabilized coal gangue mixture (M100 group) were compared, and the influence of different particle size coal gangue aggregates into the mixture on its stiffness was discussed and the results are shown in Figure 5.

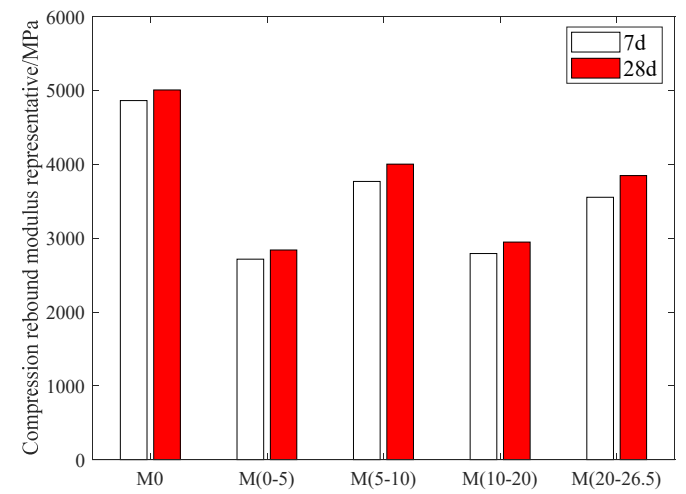

Figure 5. Compression rebound modulus of cement-stabilized coal gangue mixture

From Figure 5, the incorporation of coal gangue reduced the stiffness of the mixture, and the compression rebound modulus of each group was reduced to different degrees, which differs from the previous test results, $\mathrm{M}(0-$ 5) The compression rebound modulus of the specimens of the group and $\mathrm{M}(10-20)$ group decreased to a larger extent, compared with the reference group $\mathrm{M} 0$ at the $7 \mathrm{~d}$ age, they lost $44.1 \%$ and $42.6 \%$, respectively, and at $28 \mathrm{~d}$, compared with the reference group M0 lost $43.3 \%$ and $41.1 \%$, respectively, and the degree of decline is much greater than that of the $\mathrm{M}(5-10)$ and $\mathrm{M}(20-26.50)$ groups, indicating that $0-5 \mathrm{~mm}$ and $10-20 \mathrm{~mm}$ coal gangues replace the natural gravel thickness of the mixture. The decrease in stiffness is more obvious. For road sections with higher rigidity requirements, the quality standard of coal gangue of this particle size should be reasonably controlled or not replaced by this particle size during construction to ensure the rigidity of the material, prevent the occurrence of cracks, and affect the construction quality.

\section{Conclusions}

(1) The maximum dry density of the mixture with coal gangue is lower than the density of the natural crushed stone water-stable mixture, and the optimal moisture content is higher than that of the natural crushed stone water-stable mixture, which is the same as the density of coal gangue. The density of coal gangue is lower than that of natural gravel; however, the water absorption rate is higher.
(2) When coal gangue was mixed with a cementstabilized crushed stone mixture, the $7 \mathrm{~d}$ and $28 \mathrm{~d}$ unconfined compressive strength, splitting strength, and compression rebound modulus of the mixture were all reduced. The mechanical properties showed a deteriorating effect.

(3) All the alternative groups of coal gangue with $4 \%$ cement content can meet the strength requirements of expressways and first-class highways.

(4) The change in coal gangue particle size has a significant impact on the mechanical properties of the mixture. After the mixture of $0-5 \mathrm{~mm}$ and $10-20 \mathrm{~mm}$ coal gangue was replaced, the mixture had unconfined compressive strength, splitting strength, and the compressive resilience modulus decreased significantly. In actual applications, to ensure the quality of the project, the quality standards of coal gangue with particle sizes of 0-5 $\mathrm{mm}$ and 10-20 $\mathrm{mm}$ should be strictly controlled or the particle size should not be used instead, to avoid the obvious decrease in the mixture's strength and splitting. The strength was too low to cause cracks.

\section{Acknowledgments}

This study was supported by the National Natural Science Foundation of China (51768058) and the Key R \& D projects of Ningxia Province of China (2021BEE03004). Their results were fundamental to this study.

\section{References}

1. Huang Di. Study on research's flow properties and model its heat transfer performance for oxygenheating $\mathrm{CaC}_{2}$ production. Master dissertation of Beijing University of Technology. 2014

2. Yang guodong,Wang feng.Experimental Study on Mixture $R$ atio of Cement Stabilized Base Material with Washed Coal Gangue. Journal of Shandong Jiaotong University. 2018, 26(03):64-70.

3. Fan Wendong. The studies of using about the integrity of the cement stable macadam. Master dissertation of Chang'an University. 2012.

4. Zhou Jie. Performance evaluation of cement stabilized recycled concrete aggregate treated with waste oil and asphalt emulsion. Master dissertation of Hunan University. 2019.

5. Base in Suzhou Area. Journal of China \& Foreign Highway. 2012, 32(03):80-85.

6. Fu Luxin. Study on Strengthening Treatment and Application of Cement Stabilized Aggregate Milling Masterial. Shandong University of Technology. 2019. 